\title{
ARTICULO ORIGINAL-2016: Características epide- miológicas y complicaciones de los pacientes operados por adenomas de hipófisis por vía transesfenoidal en- doscópica en la Unidad de Cirugía de Base de Cráneo del Hospital México
}

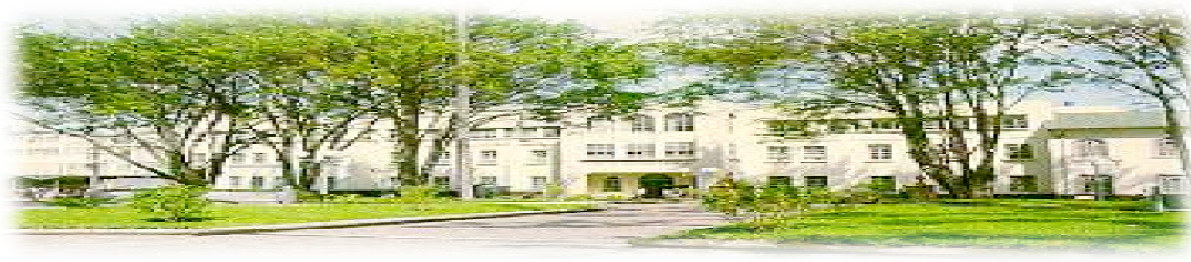

Hospital San Juan de Dios, San José, Costa Rica. Fundado en 1845
ISSN

2215-2741

Recibido: 22/05/15

Aceptado: 02/11/15

\author{
Emmanuelle Vargas Valenciano ${ }^{1}$ \\ Miguel Ángel Esquivel Miranda ${ }^{2}$ \\ Andrés Obando Valverde ${ }^{3}$ \\ Mónica Quiroga Galindo ${ }^{4}$
}

${ }^{1}$ Médico Residente de Neurocirugía, Hospital México, Caja Costarricense de Seguro Social, correo electrónico: emanuelvv14@gmail.com

${ }^{2}$ Médico Especialista de Neurocirugía, Hospital México, Caja Costarricense de Seguro Social

${ }^{3}$ Médico Especialista en Otrorrinolaringología, Hospital México, Caja Costarricense de Seguro Social

${ }^{4}$ Médico Especialista de Neurocirugía, Hospital México, Caja Costarricense de Seguro Social

\section{RESUMEN}

En el Hospital México, desde el año 2009 se constituyó una unidad de cirugía endoscópica de la base de cráneo que desde un abordaje multidisciplinario permite la valoración y tratamiento de estos pacientes. En el presente estudio se documentaron un total de 47 pacientes durante los años 2012 y 2013 presentando una edad promedio de 49 años, siendo de predominio femenino. Dentro de la cohorte, la mayoría de los adenomas se catalogaron como no funcionantes (70\%), seguido de productores de GH $(15 \%)$ y ACTH (15\%). Aproximadamente la mitad de los casos presentaron déficit visual campimétrico y $25 \%$ tenían antecedente de cirugía previa por esa misma lesión. Se operaron $13 \%$ microadenomas, $74 \%$ macroadenomas y $13 \%$ adenomas gigantes. La duración promedio del procedimiento fue de 247 minutos. El porcentaje de fístula postoperatoria fue de $1,5 \%$. No se presentaron muertes en esta serie. A pesar de que se documentó un porcentaje ligeramente mayor del $10 \%$ de insuficiencia suprarrenal y diabetes insípida postoperatoria la mayoría de estos casos presentaban esta condición de previo. 


\section{PALABRAS CLAVE}

Tumor cerebral, neurocirugía, endocrinología, neurología, transesfenoidal

\section{ABSTRACT}

In the last years there has been a change in the surgery treatment of the sellar and parasellar pathology with the transnasaltransphenoidal endoscopic approach being actually the mainroute for the treatment of most of these lesions. In the Hospital Mexico, there is an endoscopic cranial base surgery unit since 2009 which from a multidisciplinary approach evaluates and treats these patients. In this article, a cohort of the patients with a transphenoidal approach for treatment of hypophysis adenomas in the 20122013 years have been revised with a total of 47 patients. The mean age was 49 years, with more prevalence in women (62\%). Most of the tumors were diagnosed as non-functional, followed by GH producers and ACTH producers. Aproximately half of the patients had a campimetry deficit and $17 \%$ had a previous surgery, most of them transcranial. $74 \%$ of the patients had macroadenomas, $13 \%$ microadenomas and 13\% giant adenomas. The mean duration of the procedure was 247 minutes. The postoperatory cerebrospinal fluid leakage percentage was $1,5 \%$. Suprarrenal insufficiency and insipid diabetes in the postoperatory period was slightly over $10 \%$, however most of the cases were present since the preoperatory setting and were transitory.

\section{KEY WORDS}

Brain tumor, neurosurgery, endocrinology, neurology, transphenoidal

\section{ABREVIATURAS}

GH: Hormona de Crecimiento

ACTH: Hormona adrenocorticotropa

PRL: Prolactina

TSH: Hormona estimulante de tiroides

FSH: Hormona folículo-estimulante

LH: Hormonaluteinizante

LCR: Líquidocefalorraquídeo

\section{INTRODUCCIÓN}

Los tumores del sistema nervioso central constituyen un porcentaje pequeño del total de tumores diagnosticados en Costa Rica, ${ }^{(1)}$ sin embargo, el impacto que éstos presentan sobre la sobrevida y especialmente sobre la calidad de vida de los pacientes es muy significativo desde el punto de vista biológico, psicológico, familiar y social. ${ }^{(2)}$

De la totalidad de tumores intracraneanos, los adenomas de hipófisis constituyen aproximadamente del 10 al $15 \%,{ }^{(2)}$ presentando éstos un comportamiento clínico, radiológico y pronóstico muy distinto del resto de los tumores intracraneanos. ${ }^{(3)}$ Parte de este comportamiento se debe a que aunque estos tumores no tienen tanta tendencia a malignizarse como otros tumores cerebrales, su proximidad con estructuras críticas provoca muchas de sus complicaciones y sintomatología. ${ }^{(3)}$ En las últimas décadas, con el desarrollo de nuevas tecnologías, los abordajes endoscópicos a la base del cráneo han tomado un gran auge y han permitido mejorar muchas de las dificultades que se presentaban con otras tecnologías y en la actualidad se consideran la terapia de elección para la resección de la mayoría de lesiones intraselares así como lesiones con extensión paraselar y supraselar. ${ }^{(4,5)}$

Actualmente, en el Hospital México existe una unidad de cirugía de base de cráneo desde el año 2009, la cual realiza las resecciones de muchos adenomas de hipófisis por la vía endoscópica transnasaltransesfenoidal. Este estudio pretende determinar las características epidemiológicas y complicaciones del período intrahospitalario de los pacientes operados en el Hospital México por la Unidad de Cirugía de Base de Cráneo por la vía transnasal transesfenoidal endoscópica.

\section{METODOLOGÍA}

Se estudió una cohorte conformada por los pacientes operados desde enero del año 2012 a diciembre del año 2013 de adenomas de hipófisis a través de un abordaje endoscópico transnasaltransesfenoidal. Los datos de los pacientes se obtuvieron a partir de la base de datos de pacientes operados de adenomas de hipófisis de la Unidad de Cirugía Endoscópica de Base de Cráneo del Hospital México, la cual se construye a partir de la información de los pacientes duran- 
te su evaluación en consulta externa y su evolución hospitalaria. Para evaluar las variables estudiadas se realizaron análisis estadísticos descriptivos, tales como frecuencias absolutas, porcentajes, medias y desviación estándar.

Tabla I. Características de los pacientes de la presente cohorte con respecto al número de pacientes, distribución por sexo, antecedente de cirugía previa, comorbilidades, tamaño del tumor, y producción hormonal del tumor

\begin{tabular}{|c|c|}
\hline Característica & Total o Porcentaje \\
\hline Número de Pacientes & 47 \\
\hline Sexo: Mujer/hombre (razón) & $29 / 18(1,6)$ \\
\hline $\begin{array}{c}\text { Antecedente de cirugía previa } \\
\text { - } \quad \text { Transcraneal } \\
\text { - Transesfenoidal }\end{array}$ & $\begin{array}{c}17 \% \text { ( } 8 \text { pacientes }) \\
5 \text { pacientes } \\
3 \text { pacientes }\end{array}$ \\
\hline $\begin{array}{l}\text { Comorbilidades } \\
\text { - Hipertensión arterial } \\
\text { - } \text { Diabetes Mellitus } \\
\text { - Asma } \\
\text { - Insuficiencia Renal } \\
\text { - Hepatopatía }\end{array}$ & $\begin{array}{c}41 \% \\
18 \% \\
2,6 \% \\
0 \% \\
5 \%\end{array}$ \\
\hline
\end{tabular}

\begin{tabular}{l} 
Tamaño \\
- Microadenoma \\
- Macroadenoma \\
- Adenoma Gigante \\
\\
No funcionante \\
Funcionante \\
- Acromegalia \\
- Cushing \\
- Prolactinoma \\
\hline
\end{tabular}

\section{Resultados}

En el presente estudio se analiza un total de 47 pacientes. En promedio la edad de los mismos fue de 49,7 años (rango 16-78 años), con una distribución por sexo de $62 \%$ de mujeres y $38 \%$ de hombres (véase Tabla 1). Aproximadamente un $41 \%$ de la población tenían como antecedente clínico hipertensión arterial, 18\% diabetes mellitus, $2,6 \%$ asma y $5 \%$ hepatopatías.

Con respecto a la distribución por tipo hormonal, la mayoría de los adenomas se catalogaron por diagnóstico histológico como adenomas no funcionantes $(70 \%)$, seguido de adenomas productores de GH (15\%) o ACTH (15\%). No se documentaron adenomas productores de TSH o PRL en esta serie. (véase Tabla I).

Aproximadamente un $17 \%$ de los pacientes presentaban un antecedente de cirugía previa por lesión selar, en su mayoría por medio de un abordaje transcraneal (62\%), siendo algunos pacientes referidos de hospitales regionales y otros propios de nuestro centro. Otro aspecto importante es que casi la mitad de los casos $(47,4 \%)$ se presentaron inicialmente con algún grado significativo de deterioro visual (en su mayoría hemianopsias o cuadrantopsias y en menor medida amaurosis).

Con respecto al tamaño, un $13 \%$ de los casos se catalogaron como microadenoma de hipófisis (menor a $1 \mathrm{~cm}$ de diámetro máximo), 74\% como macroadenomas (1-4 $\mathrm{cm}$ diámetro máximo) y $13 \%$ como adenomas gigantes (mayor a $4 \mathrm{~cm}$ de diámetro máximo) (véase Tabla I y Figura 1). Por otra parte, con respecto a las complicaciones transoperatorias, durante la realización del procedimiento quirúrgico se documentó salida de líquido cefalorraquídeo transoperatoria en un $21,7 \%$ de los casos, la cual disminuyó de forma importante en el postoperatorio $(1,5 \%$ de los casos). Otras complicaciones transoperatorias fueron sangrado del seno cavernoso en $1,1 \% \mathrm{y}$ bradicardia transoperatoria $1,1 \%$.

Se catalogó como resección macroscópica completa, de acuerdo a la valoración propia del cirujano de forma transoperatoria, en un $65 \%$ de los casos. Con respecto al tiempo quirúrgico, se documentó una duración promedio de 247 minutos, con un total de días de internamiento prequirúrgico de 1,2 días y postoperatorios de 5,6 días, para un total de días de internamiento 7,7 días.

Finalmente, con respecto a la evolución postoperatoria, se presentó insuficiencia suprarrenal en $13,5 \%$, diabetes insípida en $10,8 \%$ sin documentar ningún caso de meningitis. Asimismo, se 
documentó la presencia de fístula postoperatoria en $1,5 \%$ de los casos.

\section{DISCUSIÓN}

Los adenomas de hipófisis son tumores usualmente benignos que se originan de las células de la adenohipófisis, $(2,5,6)$ presentando su crecimiento dentro de una pseudocápsula que los separa del tejido glandular normal adyacente. ${ }^{(3)}$ Estos tumores presentan su sintomatología debido a su compresión del tejido normal adyacente (comprimiendo la vía visual o el tejido glandular hipofisiario principalmente) o por la hiperproducción de alguna hormona hipofisiaria. Se han desarrollado numerosas clasificaciones para los adenomas de hipófisis, siendo las principales la clasificación funcional y la clasificación por tamaño.

La edad de presentación más frecuente es entre los 30 y 60 años (con un promedio de 46 años), (2) presentando una predominancia ligera en el sexo femenino, ${ }^{(9)}$ especialmente en edades más tempranas. (2) La mayoría de los casos se deben a un origen esporádico siendo la minoría los de tipo familiar. ${ }^{(2)}$ En el caso de nuestra serie, la edad promedio obtenida fue de 49 años, la cual se encuentra cercana al promedio reportado en la literatura. De la misma forma, se logró documentar un predominio del sexo femenino en nuestra muestra (62\% sexo femenino y $38 \%$ masculino), concordando de la misma forma con otras series reportadas en la literatura.

Desde el punto de vista funcional, los adenomas de hipófisis se clasifican de acuerdo al patrón de producción hormonal tumoral. En las series quirúrgicas reportadas a nivel mundial, son más frecuentes los adenomas de tipo clínicamente no funcionante (incluyen aquellos con células no productoras y los constituidos por células productoras de FSH o LH, los cuales presentan una secreción hormonal deficiente por lo que se presentan usualmente sin manifestaciones clínicas importantes). En nuestro estudio, que corresponde a pacientes operados (es una serie quirúrgica, no la totalidad de casos de adenomas de hipofisis diagnosticados), la mayoría de los casos se catalogaron como adenomas no funcionantes $(70 \%)$, siendo este porcentaje mayor a los promedios reportados en otras latitudes (30-55\% en las distintas series). $(5,10)$

Figura 1. Ejemplos de casos de adenomas de hipó-
fisisde acuerdo a la clasificación por diámetro máximo tumoral

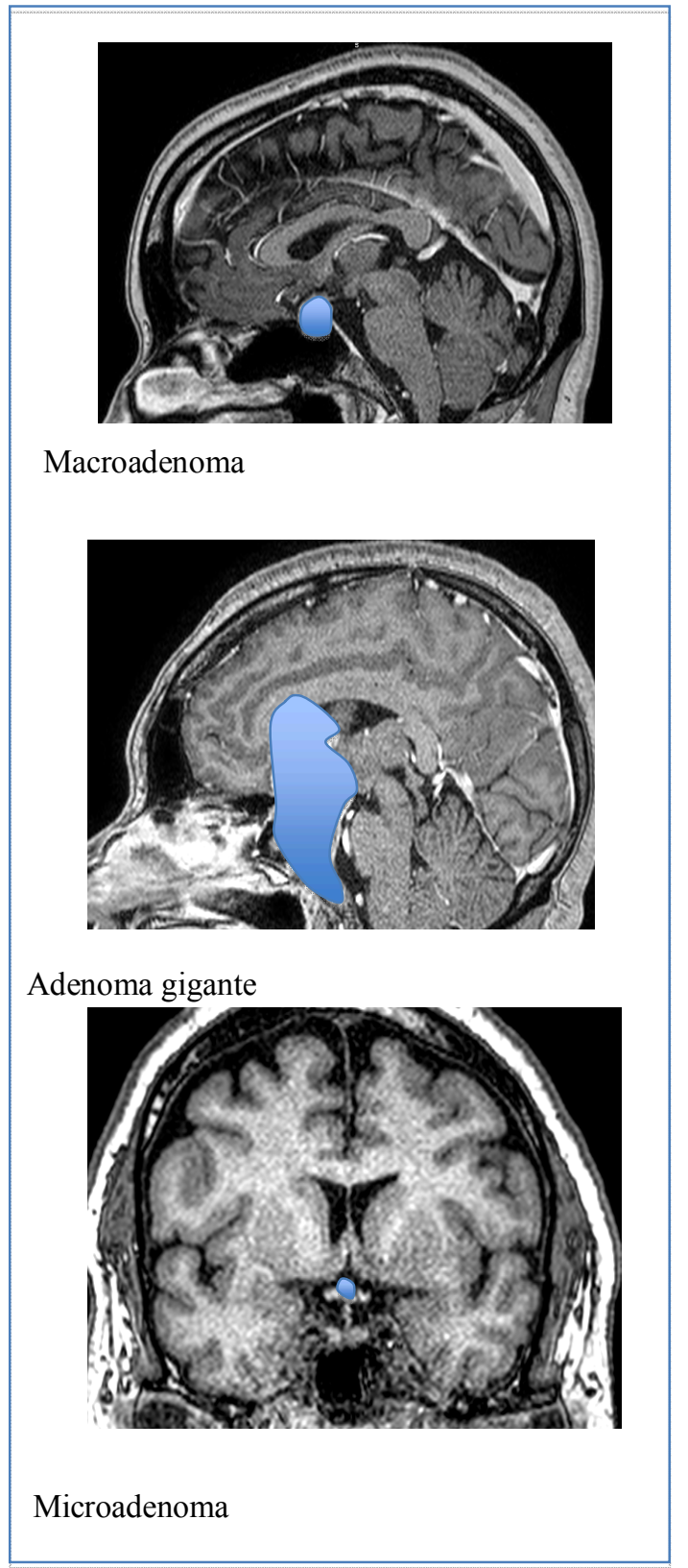


El segundo tipo hormonal más frecuente en nuestra serie son los adenomas productores de $\mathrm{GH}$ $(15 \%)$ y ACTH (15\%), con una proporción semejante a lo esperado de acuerdo a la literatura $\left(17 \%\right.$ y $12 \%$ respectivamente $\left.{ }^{5,10}\right)$. Finalmente, en nuestra serie no se documentó tumores productores de prolactina, lo cual es inusual con respecto a las distintas series quirúrgicas reportadas (12$30 \%$ del total corresponden a este tipo de tumor) $(5,10)$. Esta disparidad entre los hallazgos obtenidos en nuestro estudio y los reportados en la literatura se deben a la poca frecuencia de cirugía endoscópica para tumores productores de prolactina, posiblemente debido al manejo médico más intensivo y la introducción de medicamentos con mayor efectividad para este tipo de tumor con la consecuente disminución de referencias de estos casos a nuestra unidad.

Con respecto al tamaño, los adenomas de hipófisis se clasifican en microadenomas, en caso de presentar un tamaño menor de $1 \mathrm{~cm}$ de diámetro máximo, macroadenomas $(1-4 \mathrm{~cm}$ diámetro máximo) y adenomas gigantes (tamaño mayor de $4 \mathrm{~cm}$ de diámetro máximo) (véase Figura 1). La gran mayoría de los adenomas de hipófisis en el momento de presentación se clasifican como macroadenomas, usualmente con la presencia de síntomascompresivos. ${ }^{(4,5)}$ La clínica predominante es usualmente la cefalea frontal y las alteraciones visuales por compresión de estructuras adyacentes, así como la hiperproducción hormonal o la hipoproducción producto de la compresión glandular. Sin embargo, hasta un 35\% de los adenomas no secretan hormonas y únicamente producen síntomas compresivos. ${ }^{(2,3)}$

En nuestro estudio se presentaron datos semejantes a los documentados en otras series, siendo la mayoría de los adenomas de hipófisis al diagnóstico catalogados como macroadenomas (74\% del total en nuestra serie y a nivel mundial aproximadamente $\left.80 \%{ }^{(5,10)}\right)$, así como presentándose aproximadamente en la mitad de los casos un deterioro visual por compresión quiasmática. Llama la atención la proporción de adenomas gigantes que se presentaron en los dos años del estudio, posiblemente debido a un diagnóstico tardío que permitió el crecimiento tumoral a esas dimensiones.

Por otra parte, llama la atención en nuestro estudio la presencia de un $17 \%$ de los casos con el antecedente de una cirugía previa, en su mayoría a través de un abordaje transcraneal $(62 \%$ del total) que había presentado una resección subtotal o un recrecimiento tumoral. Estos datos corresponden a un posible traslape entre la predominancia de técnicas quirúrgicas abiertas hacia las técnicas endoscópicas transnasales que se ha venido desarrollando en los últimos años, siendo actualmente más frecuente que las persistencias de lesión tumoral sean abordadas a través de la vía endoscópica. ${ }^{(8)}$ En otros sitios, el porcentaje de cirugías por una persistencia o recrecimiento luego de una primera intervención es de aproximadamente $10-20 \%$ del total de las intervenciones. ${ }^{(9,10,11)}$

El tiempo quirúrgico total en esta serie fue de 247 minutos, siendo necesario un período mayor para lograr determinar si existen cambios significativos en este aspecto conforme va avanzando la experiencia de la unidad. Otro aspecto a tomar en cuenta es la utilización de algunos dispositivos intraoperatorios los cuales han permitido realizar el procedimiento quirúrgico con mayor grado de seguridad y permiten una resección mayor en tumores más complejos. En nuestro caso, la unidad cuenta con un aspirador ultrasónico que permite resecar tumores con una consistencia dura y de ubicación compleja de forma más eficiente así como un neuronavegador que ha permitido la localización intraoperatoria en tiempo real con una mayor facilidad para ubicar estructuras vasculares y nerviosas evitando de esta forma dañarlas. En conjunto, estos avances tecnológicos han permitido abordar tumores técnicamente más complejos que presentan invasión paraselar o supraselar importantes con una mayor seguridad.

\section{Complicaciones}

La cirugía endoscópica transnasaltranshipofisiaria para la resección de adenomas de hipófisis presenta complicaciones propias del procedimiento así como complicaciones médicas asociadas a la resección de patologías selares. Como complicaciones del procedimiento, en nuestra serie se documentó la salida de líquido cefalorraquídeo transoperatorio en $21,7 \%$ de los casos, situación relacionada con el procedimiento quirúrgico mismo y que actualmente ya no se considera una complicación como tal y es muy frecuente en abordajes más extensos. Sin embar- 
go, una vez transcurrido el procedimiento quirúrgico se documentó fístula de LCR postoperatoria únicamente en $1,5 \%$, valor dentro del rango normal en otros centros $(0-2 \%){ }^{(13,14)} \mathrm{Se}$ presentaron otras complicaciones menos comunes, como sangrado del seno cavernoso y bradicardia transoperatoria en un único paciente, lo cual se ha descrito como complicaciones infrecuentes en este procedimiento en tumores con gran extensión a la región paraselar. El manejo de la fístula de líquido cefalorraquídeo usualmente cuando se presenta de forma transoperatoria se trata por medio de la colocación de sustitutos de duramadre junto con material hemostático (surgicel, gelfoam, helitene), la colocación de un colgajo nasoseptal o un taponamiento con sonda Foley o Merocel ${ }^{\circledR}$. En ocasiones no se logra resolver el proceso fistuloso y requiere una reintervención para reparación del defecto.

Como complicaciones postoperatorias, en caso de documentar fístula de líquido cefalorraquídeo postoperatoria inicialmente se brindaba tratamiento con medidas antifístula tales como acetazolamida, reposo absoluto, laxantes, derivación externa y en caso necesario se realizaba una reparación quirúrgica, sin embargo, recientemente a nivel mundial se ha modificado este enfoque presentando las fístulas de líquido cefalorraquídeo como padecimiento que requiere una intervención urgente debido a sus potenciales complicaciones. Sería interesante en un futuro lograr determinar en otro estudio si existieron diferencias en cuanto a la tasa de meningitis postoperatoria entre ambos enfoques. Otra de las posibles complicaciones postoperatorias de este tipo de abordajes es la diabetes insípida, la cual se presenta aproximadamente en $0,4-17 \%$ (en promedio aproximadamente $10 \%$ de los casos) siendo en nuestra serie de 10,8\% , sin embargo, la mayoría de éstas de forma transitoria. De la misma forma se logró constatar insuficiencia suprarrenal en $13,5 \%$ de los casos, sin embargo, la mayoría de éstos presentaban esta condición desde previo al procedimiento por la compresión tumoral o por secuela de un procedimiento previo. Otras complicaciones reportadas en la literatura que no se constataron en nuestra serie son la hiponatremia (4\%) así como otros menos comunes como la meningitis $(1 \%)$, hematomas intraselares 0 muerte. ${ }^{(10-14)}$

\section{CONCLUSIÓN}

Los adenomas de hipófisis son tumores benignos histológicamente sin embargo, con una gran cantidad de complicaciones asociadas a su crecimiento y la compresión que producen en las distintas estructuras de la región selar y paraselar. En el presente estudio se evidenciaron distintos datos epidemiológicos y de complicaciones tempranas del abordaje endoscópico transnasal en una cohorte de pacientes operados por la Unidad de Cirugía de Base de Cráneo Endoscópica durante los años 2012-2013. Se logró documentar que la mayoría de las variables tienen un comportamiento semejante al reportado en otros sitios. Una de las variables que presentó un comportamiento distinto a otros sitios fue el porcentaje de prolactinomas operados, el cual es menor en nuestro centro, posiblemente debido al manejo médico intensivo que se brinda a los mismos.

\section{BIBLIOGRAFÍA}

1. Instituto Nacional de Estadística y Censos. Total de Defunciones por grupo de edades, según grandes grupos de causas de muerte y sexo. 2010. San José, Costa Rica

2. Ortiz-Plata A et al. Chapter 5: Pituitary adenomas - clinicopathological, immunohistochemical and ultrastructuralstu$d y$ en el libro Rahimi-Movaghar, V. Pituitary Adenomas. Editorial InTech. 2012

3. DeMonte F Gilbert M Mahajan A McCutcheon I. Tumors of theBrain and Spine. Editorial SpringerScienceBusiness. 2007: 191-220

4. Tabaee A et al. Endoscopic pituitary surgery: a systematic review and metaanalysis. J Neurosurg 111: 545-554, 2009

5. Jagannathan $\mathrm{J}$ et al. Benign Brain Tumors: Sellar/Parasellar Tumors. NeurolClin 25 (2007) 1231-1249

6. Rojas D Palma A Wohllk N. Manejo de los adenomas hipofisiarios. RevChilNeuro-Psiquiat 2008; 46 (2): 140-147

7. Chandler W Barkan A. Treatment of Pituitary Tumors: a Surgical Perspective. EndocrinolMetabClin N Am 37 (2008) 51-66 
8. Cappabianca P et al. Endoscopic endonasal transsphenoidal surgery in recurrent and residual pituitary adenomas: technical note.MinimInvasiveNeurosurg. 2000 Mar; 43(1):38-43

9. Rudnik A et al. Endoscopic transsphenoidal treatment in recurrent and residual pituitary adenomasfirstexpe-

rience.MinimInvasiveNeurosurg. 2006 Feb; 49(1):10-4

10. Dehdashti A. Endoscopic endonasal approach for pituitary adenomas: early surgical results in 200 patients and comparison with previous microsurgical series. Neurosurgery 2008; 62:10061017

11. Cavallo L et al. Endoscopic endonasal transsphenoidal removal of recurrent and regrowing pituitary adenomas: experience on a 59-patient series.WorldNeurosurg. 2013; 80 (3): 342-350

12. Dharambir S; Jern-Lin L. Endoscopic Pituitary Surgery. OtolaryngolClin N Am 39 (2006) 563-583

13. Senior B et al. Minimally Invasive Pituitary Surgery. Laryngoscope, 118 (2008): 1842-1855

\section{CONFLICTO DE INTERÉS Y/O AGRADE- CIMIENTOS}

Los autores declaran que no existió ningún conflicto de interés en el presente reporte. 